\title{
Pollen Morphology of Tribes Gnaphalieae, Helenieae, Plucheeae and Senecioneae (Subfamily Asteroideae) of Compositae from Egypt
}

\author{
Ahmed Kamal El-Deen Osman \\ Faculty of Science, Botany Department, South Valley University, Qena, Egypt. \\ Email: ahmosman2000@yahoo.com \\ Received October $10^{\text {th }}, 2010$; revised December $9^{\text {th }}, 2010$; accepted December $20^{\text {th }}, 2010$.

\begin{abstract}
POLLEN morphology of twenty five species representing 12 genera of tribes Gnaphalieae, Helenieae, Plucheeae and Senecioneae (Asteroideae: Asteraceae) was investigated using light and scanning electron microscopy. The genera are Phagnalon, Filago, Gnaphalium, Helichrysum, Homognaphalium, Ifloga, Lasiopogon, Pseudognaphalium, Flaveria, Tagetes, Sphaeranthus and Senecio. Two pollen types were recognized viz. Senecio pollen type and Filago pollen type. Description of each type, a key to the investigated taxa as well as LM and SEM micrographs of pollen grains are provided.
\end{abstract}

Keywords: Pollen, Morphology, Asteroideae, Asteraceae, Egypt

\section{Introduction}

Gnaphalieae, Helenieae, Plucheeae and Senecioneae (Asteroideae: Asteraceae) are of the well represented tribes in Egypt, where 12 genera with about thirty five species are native in the country [1]. Reference [2] separated the Compositae pollen into two groups based on the gross morphology of the pollen grain: the liguliflorae-type characterized by echinolophate pollen, which is generally found in the Cichorieae; and the tubuliflorae- type, which is found in many other tribes. Reference [3] recognized three pollen types, i.e., psilate (this type can not be easily recognized), echinate, and lophate pollen. Based on pollen wall ultrastructure, [4] and [5] reconsidered these pollen types and recognized five major pollen wall types, the Helianthoid, the Senecioid, the Arctotoid, the Anthemoid and pollen types without designated patterns. These pollen types are variously distributed among the Cichorioideae and the Asteroideae. Pollen of the Barnasioideae is similar to the echinolophate pollen of the liguliflorae-type, but lacks the spines [6]. Reference [7] observed that the pollen grains of some Compositae tribes consist of three compound apertures, each one of them is made up of ecto-, meso- and endo-aperture, the apertural membrane is formed by a foot layer and endexine with complete ectexine granules, the ectoaperture involves the tectum and infratectum, the mesoaper- ture involves the foot layer and the outer layer of the endexine and the endoaperture involves the inner layer of the endoxine. The intine is thickened considerably in Anthemideae near the aperture. Reference [8] described Gnaphalieae pollen as a two-layered pollen sexine with an outer baculae and an inner perforated layer. Reference $[9,10]$ observed that the pollen morphology and anatomy of the Plucheeae correspond to that of the Inuleae; thus, the pollen is echinate and caveate with one layer of baculae between the spines and two baculate layer in the spines bases. This paper considers pollen grains of the tribes Gnaphalieae, Helenieae, Plucheeae and Senecioneae in the flora of Egypt, which may prove of value in systematic treatments. Pollen types that show all the possible characteristic features (shape, size, apertures, wall stratification) are considered, with special reference to the specific characters of each pollen type. The available palynological data will be discussed.

\section{Materials and Methods}

Pollen materials were removed from herbarium specimens identified according to $[1,11,12]$ and [13]. The specimens were kept in Cairo University Herbarium (CAI) and Herbarium of South Valley University (QNA, proposed abbreviation) (Table 1). Light microscopy (LM) observations were carried out on acetolyzed pollen and 
Table 1. Investigated specimens of 25 species (12 genera) of Gnaphalieae, Helenieae, Plucheeae \& Senecioneae (Asteraceae) according to [14].

\begin{tabular}{|c|c|c|}
\hline Species & Collector & Herb. \\
\hline \multicolumn{3}{|l|}{ Subfamily Asteroideae } \\
\hline \multicolumn{3}{|l|}{ I-Tribe Gnaphalieae } \\
\hline \multicolumn{3}{|l|}{ 1. Unassigned Gnaphalieae } \\
\hline Phagnalon barbeyanum & EL-GARF \& A.K.OSMAN, s.n. & QNA \\
\hline P. nitidum & FAYED et al. s.n. & QNA \\
\hline P. rupestre & EL-GARF\& A.K.OSMAN, s.n. & QNA \\
\hline P. schweinfurthii & A. K. OSMAN \& K.N. ABDEL KHALIK, s.n. & CAI \\
\hline \multicolumn{3}{|l|}{ 2.Subtribe Gnaphaliinae } \\
\hline Filago contracta & EL-GARF \& A.K.OSMAN, s.n. & CAI \\
\hline F. desertorum & EL-GARF \& A.K.OSMAN, s.n. & QNA \\
\hline F. mareotica & EL-GARF \& A.K.OSMAN, s.n. & QNA \\
\hline F. prolifera & A. K. OSMAN \& K.N. ABDEL KHALIK, s.n. & CAI \\
\hline Gnaphalium uliginosum & A. K. OSMAN, s.n. & CAI \\
\hline Helichrysum conglobatum & EL-GARF \& A.K.OSMAN, s.n. & CAI \\
\hline H. glumaceum & A. K. OSMAN \& K.N. ABDEL KHALIK, s.n. & CAI \\
\hline H. orientale & EL-GARF \& A.K.OSMAN, s.n. & CAI \\
\hline Homognaphalium pulvinatum & A. K. OSMAN, s.n. & CAI \\
\hline Ifloga spicata & (Forssk.) Sch.Bip. FAYED et al. s.n. & QNA \\
\hline Lasiopogon muscoides & (Desf.) DC. EL-GARF \& A.K.OSMAN, s.n. & CAI \\
\hline Pseudognaphalium luteoalbum & EL-GARF \& A.K.OSMAN, s.n. & CAI \\
\hline \multicolumn{3}{|l|}{ II-Tribe Helenieae } \\
\hline \multicolumn{3}{|l|}{ 1.Subtribe Flaveriinae } \\
\hline Flaveria bidentis & EL-GARF \& A.K.OSMAN, s.n. & CAI \\
\hline \multicolumn{3}{|l|}{ 2.Subtribe Pectidinae } \\
\hline Tagetes minuta & A. K. OSMAN, s.n. & QNA \\
\hline \multicolumn{3}{|l|}{ III-Tribe Plucheeae } \\
\hline Sphaeranthus suaveolens & EL-GARF \& A.K.OSMAN, s.n. & CAI \\
\hline \multicolumn{3}{|l|}{ IV-Tribe Senecioneae } \\
\hline \multicolumn{3}{|l|}{ 1.Subtribe Senecioninae } \\
\hline Senecio aegyptius & A. K. OSMAN, s.n. & CAI \\
\hline S. flavus & FAYED et al. s.n. & QNA \\
\hline S. glaucus & subsp. coronopifolius EL-GARF \& A.K.OSMAN, s.n. & QNA \\
\hline S. glaucus & subsp. glaucus EL-GARF \& A.K.OSMAN, s.n. & CAI \\
\hline S. hoggariensis & A. K. OSMAN \& K.N. ABDEL KHALIK, s.n. & CAI \\
\hline S. vulgaris & EL-GARF \& A.K.OSMAN, s.n. CAI & \\
\hline
\end{tabular}

$\mathrm{CAI}=$ Cairo University Herbarium, QNA = Qena University Herbarium (proposed name), s.n. = collecting number is missing. 
prepared according to the method of [15] and SEM observations were made on acetolyzed grains coated with a thin layer of gold/palladium for 3 minutes using a EMITECH K550 sputter according to the Erdtman method [16]. SEM examined with a JEOL-6300 SEM of Central Lab., Faculty of Science, South Valley University, Qena, Egypt. The terminology used is that of $[17,18]$ and [19].

\section{Results}

The subtribes, genera and species of tribes Gnaphalieae, Helenieae, Plucheeae and Senecioneae (according to [14]) that are represented in the flora of Egypt are arranged alphabetically to facilitate consultation. For each species, the valid scientific name is given followed by the citation of the authority and the date of publication. Synonymy is at a minimum to avoid complications. For full synonymy of the species see [1,12] and [13].

A careful examination of the available pollen material of the Egyptian species of tribes Gnaphalieae, Helenieae, Plucheeae and Senecioneae revealed the presence of 2 pollen types, which can be distinguished through the following key.

1.a. Pollen grains 3-zonocolpororate..Senecio pollen type

b. Pollen grains 3-zonocolporate..........Filago pollen type Main characters of pollen types.

1. Filago pollen type.

LM Figures 1(i)-(p), Figures 2(a), (b), (i), (j), (m-p). SEM Figures 3(i)-(m), Figures 4(e)-(i). (Tables 2, 3).

Pollen grains 3-colporate, $(\mathrm{NPC}=345)$, spheroidal to prolate-sheroidal $(\mathrm{P} / \mathrm{E}=1.00-1.09), 20-24(18-25) \times 20-23$ (19-25) $\mu \mathrm{m}$. Apocolpium diameter 5-7 (3-8) $\mu \mathrm{m}$. Colpi 13-16 (12-18) $\mu \mathrm{m}$ long, 2-4 (2-5) $\mu \mathrm{m}$ slightly wide, wide or slender at the equator, pointed or tapered towards the ends. Mesocolpia 12-16 (10-18) $\mu \mathrm{m}$ wide. Ora lolongate (circular only in Homognaphalium pulvinatum) with elleptic to ovate-shaped, 4-6 (3-8) $\mu \mathrm{m}$ in diameter. Exine 3-5 (2-6) $\mu \mathrm{m}$ thick (spinules included) at centre of mesocolpia, decreasing slightly in thickness towards the aperture margins. Sexine as thick as nexine or thicker than nexine in Gnaphalium uliginosum and Lasiopogon muscoides, tectate, has sharp spinules (spines in Gnaphalium uliginosum) with broad bases, 1-3 (1-4) $\mu \mathrm{m}$ high, perforated; sculpturing diameter the same on the whole of the pollen surface or occasionally restrict to the echinae bases that beset all the pollen surface (perforation increases towards the aperatures margins inGnaphalium uliginosum); the intratectal elements thin, minute, radial and supported by fairly thin and short infratectal columellae. Nexine 1-2 (1-3) $\mu \mathrm{m}$ thick, increasing in thickness towards the aperture margins.

The following taxa belong to this type:

I-Tribe Gnaphalieae
Subtribe Gnaphaliinae Less.

Genus Filago L. Syn. Evax Gaertn (4 species).

Species included:

1. F. contracta (Boiss.) Chrtek \& Holub, Preslia 45: 3 (1963).

Syn. Evax contracta Boiss., Diagn. Pl. Orient., ser. 1, 11: 3 (1849).

2. F. desertorum Pomel, Nouv. Mat. Fl. Atlant. 1: 46 (1874).

Syns. Filago spathulata C. Presl forma desertorum (Pomel) Pamp., Atti R. Ist. Ven. Sci. Lett. Arti 92: 234 (1932).

Evax mauritanica Pomel var. cyrenaica Pamp., Nuovo Giorn. Bot. Ital., n. s., 34: 962 (1927).

3. F. mareotica Delile, Descr. Egypte, Hist. Nat. 274, t. 47, f. 2 (1814).

Syns. Filago mareotica Delile var. floribunda (Pomel) Maire, Bull. Soc. Hist. Nat. Afr. Nord 26: 209 (1935)

Gifolaria mareotica (Delile) Chrtek \& Holub, Preslia 35: 10 (1963).

4. F. prolifera Pomel, Nouv. Mat. Fl. Atlant. 1: 47 (1874).

Syns. Filago germanica L. subsp. prolifera (Pomel) Maire, Cat. Pl. Maroc 3: 746 (1934).

Filago prolifera Pomel subsp. taeckholmiana Chrtek in Tackh., Stud. Fl. Egypt, ed. 2, 554 (1974).

Genus Gnaphalium L. Syns. Amphidoxa DC., Filaginella Opiz (one species).

Species included:

5. G. uliginosum L., Sp. Pl., ed. 1, 856 (1753).

Syn. Filaginella uliginosa (L.) Opiz, Abh. Bohm. Ges. Wiss., ser. 5, 8 (Sitzungsber. Sect.): 52 (1854).

Genus Homognaphalium Kirp. Syn. Homognaphalium fayed \& Zareh non Kirp (one species).

Species included:

6. H. pulvinatum (Delile) Fayed \& Zareh, Willdenowia 18: 451 (1989).

Syn. Gnaphalium pulvinatum Delile, Descr. Egypte, Hist. Nat. 266, t. 44 (1814).

Genus Lasiopogon Cass (one species).

Species included:

7. L. muscoides (Desf.) DC., Prodr. 6: 246 (1838).

Syn. Gnaphalium muscoides Desf., Fl. Atlant. 2: 267, t. 231 (1799).

Genus Pseudognaphalium Kirp. Syn. Hypelichrysum Kirp (one species).

Species included:

8. P. luteoalbum (L.) Hilliard \& B. L. Burtt, Bot. J. Linn. Soc. 82: 206 (1981).

Syn. Gnaphalium luteoalbum L., Sp. P1., ed. 1, 851 (1753) 

of Compositae from Egypt

Table 2. Tabular summary showing the Pollen Grains Dimensions $(\mu \mathrm{m})$.

\begin{tabular}{|c|c|c|c|c|c|c|c|c|c|c|c|}
\hline Species & P. & E. & P/E. & $\begin{array}{l}\text { Ex. th. Incl. } \\
\text { echin. }\end{array}$ & $\begin{array}{l}\text { Ech. } \\
\text { Len. }\end{array}$ & $\begin{array}{l}\text { Nex. } \\
\text { Th. }\end{array}$ & Colp. Len. & $\begin{array}{l}\text { Colp. } \\
\text { wid. }\end{array}$ & $\begin{array}{l}\text { Ora. } \\
\text { diam. }\end{array}$ & Apo. diam. & $\begin{array}{l}\text { Meso. } \\
\text { diam. }\end{array}$ \\
\hline \multicolumn{12}{|l|}{ I-Tribe Gnaphalieae } \\
\hline $\begin{array}{l}\text { 1. Unassigned } \\
\text { Gnaphalieae }\end{array}$ & & & & & & & & & & & \\
\hline $\begin{array}{l}\text { Phagnalon } \\
\text { barbeyanum }\end{array}$ & $28(25-31)$ & $27(25-30)$ & 1.04 & $5(4-6)$ & $3(2-4)$ & $2(1-3)$ & $16(15-18)$ & $5(4-6)$ & $8(7-9)$ & $8(7-9)$ & $17(15-18)$ \\
\hline P. nitidum & $25(20-30)$ & $27(25-30)$ & 0.93 & $5(3-6)$ & $2(2-3)$ & $2(1-3)$ & $13(12-15)$ & $5(3-7)$ & $8(7-9)$ & $8(7-9)$ & $12(10-13)$ \\
\hline P. rupestre & $32(30-35)$ & $28(26-35)$ & 1.10 & $5(3-7)$ & $3(2-4)$ & $2(1-3)$ & $15(13-17)$ & $5(4-6)$ & $8(5-10)$ & $8(7-10)$ & $17(15-20)$ \\
\hline P. schweinfurthii & $27(23-28)$ & $29(25-33)$ & 0.93 & $5(3-7)$ & $3(2-4)$ & $1(1-2)$ & $15(13-17)$ & $5(4-6)$ & $7(6-8)$ & $10(8-12)$ & $15(12-16)$ \\
\hline \multicolumn{12}{|l|}{$\begin{array}{l}\text { 2.Subtribe } \\
\text { Gnaphaliinae }\end{array}$} \\
\hline Filago contracta & $21(20-23)$ & $21(20-23)$ & 1.00 & $3(2-4)$ & $1(1-2)$ & $2(1-3)$ & $15(13-17)$ & $4(2-5)$ & $5(4-6)$ & $7(5-8)$ & $12(10-15)$ \\
\hline F. desertorum & $23(20-25)$ & $23(20-25)$ & 1.00 & $3(2-4)$ & $1(1-2)$ & $1(1-2)$ & $16(15-18)$ & $3(3-4)$ & $5(4-6)$ & $6(5-8)$ & $13(12-15)$ \\
\hline F. mareotica & $24(22-25)$ & $22(20-25)$ & 1.09 & $3(2-4)$ & $1(1-2)$ & $2(1-3)$ & $16(15-18)$ & $4(3-5)$ & $6(5-8)$ & $7(5-8)$ & $15(12-18)$ \\
\hline F. prolifera & $23(20-25)$ & $23(20-25)$ & 1.00 & $4(2-5)$ & $1(1-2)$ & $2(1-3)$ & $16(15-18)$ & $4(2-5)$ & $5(3-7)$ & $5(3-7)$ & $16(15-18)$ \\
\hline $\begin{array}{l}\text { Gnaphalium } \\
\text { uliginosum }\end{array}$ & $21(20-23)$ & $21(20-23)$ & 1.00 & $5(4-6)$ & $3(2-4)$ & $1(1-2)$ & $13(12-15)$ & $2(2-3)$ & $4(2-5)$ & $5(3-7)$ & $12(10-13)$ \\
\hline $\begin{array}{l}\text { Helichrysum } \\
\text { conglobatum }\end{array}$ & $21(20-23)$ & $21(20-23)$ & 1.00 & $3(2-4)$ & $2(1-3)$ & $1(1-2)$ & $13(12-15)$ & $3(2-4)$ & $5(3-7)$ & $8(7-9)$ & $13(12-14)$ \\
\hline H. glumaceum & $26(25-28)$ & $26(25-28)$ & 1.00 & $5(4-6)$ & $3(2-4)$ & $2(2-3)$ & $18(15-20)$ & $4(3-5)$ & $5(3-6)$ & $5(4-6)$ & $14(12-15)$ \\
\hline H. orientale & $26(25-28)$ & $28(25-30)$ & 0.93 & $5(4-6)$ & $3(2-4)$ & $2(1-3)$ & $12(10-15)$ & $4(3-5)$ & $6(5-7)$ & $7(5-8)$ & $15(13-17)$ \\
\hline $\begin{array}{l}\text { Homognaphalium } \\
\text { pulvinatum }\end{array}$ & $22(20-25)$ & $22(20-25)$ & 1.00 & $3(2-4)$ & $2(1-3)$ & $1(1-2)$ & $13(12-15)$ & $4(3-5)$ & $5(4-6)$ & $7(5-8)$ & $13(12-15)$ \\
\hline Ifloga spicata & $21(20-23)$ & $21(20-23)$ & 1.00 & $4(3-5)$ & $2(1-3)$ & $1(1-2)$ & $13(12-15)$ & $3(2-4)$ & $4(3-5)$ & $7(5-8)$ & $11(9-15)$ \\
\hline $\begin{array}{c}\text { Lasiopogon } \\
\text { muscoides }\end{array}$ & $20(19-23)$ & $20(19-23)$ & 1.00 & $3(2-4)$ & $1(1-2)$ & $1(1-2)$ & $13(12-14)$ & $3(2-5)$ & $5(4-6)$ & $5(3-6)$ & $12(10-13)$ \\
\hline $\begin{array}{l}\text { Pseudognaphalium } \\
\text { luteoalbum }\end{array}$ & $21(18-22)$ & $21(19-22)$ & 1.00 & $3(2-4)$ & $2(1-3)$ & $1(1-2)$ & $13(12-15)$ & $3(2-4)$ & $4(3-5)$ & $5(3-7)$ & $12(10-13)$ \\
\hline \multicolumn{12}{|l|}{ II-Tribe Helenieae } \\
\hline \multicolumn{12}{|l|}{$\begin{array}{l}\text { 1.Subtribe } \\
\text { Flaveriinae }\end{array}$} \\
\hline Flaveria bidentis & $27(25-33)$ & $31(30-33)$ & 0.87 & $7(6-8)$ & $5(4-6)$ & $2(1-3)$ & $16(15-18)$ & $4(3-5)$ & $7(5-8)$ & $6(5-8)$ & $17(15-20)$ \\
\hline \multicolumn{12}{|l|}{$\begin{array}{l}\text { 2.Subtribe } \\
\text { Pectidinae }\end{array}$} \\
\hline Tagetes minuta & $44(42-45)$ & $44(42-45)$ & 1.00 & $8(7-9)$ & $5(4-6)$ & $2(2-4)$ & $21(20-25)$ & $5(3-6)$ & $8(7-9)$ & $16(15-18)$ & $24(22-25)$ \\
\hline \multicolumn{12}{|l|}{ III-Tribe Plucheeae } \\
\hline $\begin{array}{l}\text { Sphaeranthus } \\
\text { suaveolens } \\
\text { IV-Tribe } \\
\text { Senecioneae }\end{array}$ & $23(22-25)$ & $23(22-25)$ & 1.00 & $4(3-5)$ & $5(3-6)$ & & $6(5-8)$ & $8(7-9)$ & $10(8-12)$ \\
\hline \multicolumn{12}{|l|}{$\begin{array}{c}\text { 1.Subtribe } \\
\text { Senecioninae }\end{array}$} \\
\hline Senecio aegyptius & $31(30-33)$ & $32(30-35)$ & 0.97 & $5(4-6)$ & $3(2-4)$ & $2(1-3)$ & $18(17-20)$ & $5(4-6)$ & $6(5-8)$ & $6(5-8)$ & $15(13-17)$ \\
\hline S. flavus & $29(27-30)$ & $29(25-33)$ & 1.00 & $6(5-7)$ & $3(2-4)$ & $1(1-2)$ & $16(15-18)$ & $5(4-6)$ & $7(5-8)$ & $6(5-10)$ & $14(12-15)$ \\
\hline $\begin{array}{l}\text { S. glaucus subsp. } \\
\text { coronopifolius }\end{array}$ & $25(23-26)$ & $25(23-26)$ & 1.00 & $5(4-6)$ & $2(2-3)$ & $1(1-2)$ & $15(14-16)$ & $4(3-5)$ & $5(4-6)$ & $5(3-6)$ & $13(12-14)$ \\
\hline $\begin{array}{l}\text { S. glaucus subsp. } \\
\text { glaucus }\end{array}$ & $31(30-33)$ & $33(31-35)$ & 0.94 & $5(4-6)$ & $4(3-5)$ & $2(2-3)$ & $19(17-20)$ & $4(3-5)$ & $5(4-6)$ & $8(7-10)$ & $14(12-15)$ \\
\hline S. hoggariensis & $37(35-38)$ & $42(40-45)$ & 0.88 & $6(5-8)$ & $3(2-4)$ & $1(1-2)$ & $27(25-30)$ & $4(3-5)$ & $8(7-9)$ & $10(8-12)$ & $18(15-20)$ \\
\hline S. vulgaris & $35(30-38)$ & $41(35-45)$ & 0.85 & $7(6-8)$ & $5(3-6)$ & $3(1-4)$ & $19(15-20)$ & $5(4-6)$ & $8(7-9)$ & $8(7-10)$ & $16(15-18)$ \\
\hline
\end{tabular}

P. $=$ Polar axis, E. = Equatorial diameter, $\mathrm{P} / \mathrm{E}=$ The ratio of the length of the polar axis $(\mathrm{P})$ to the equatorial diameter $(\mathrm{E})$, Ech. Len. $=$ echinae length, Ex. Th. incl. echin. = exine thick includes echinae, Nex. Th. = nexine thick, Colp. Len. = colpus length, Colp. wid. = colpus width, Ora. diam. $=$ ora diameter, Apo. diam. $=$ apocolpium diameter, Meso. diam. $=$ mesocolpium diameter, $\mu \mathrm{m}=$ micrometer. 
Table 3. Tabular summary showing the Description of LM \& SEM Samples.

\begin{tabular}{|c|c|c|c|c|c|c|c|c|c|c|}
\hline Species & $\begin{array}{l}\text { Pollen } \\
\text { Class }\end{array}$ & $\begin{array}{l}\text { Pollen } \\
\text { Shape }\end{array}$ & $\begin{array}{l}\text { Col. wid. } \\
\text { at eq. }\end{array}$ & $\begin{array}{l}\text { Colpi } \\
\text { Ends }\end{array}$ & Ora Shape & $\begin{array}{l}\text { Echinae } \\
\text { Type }\end{array}$ & $\begin{array}{l}\text { Sculpture } \\
\text { Type }\end{array}$ & $\begin{array}{l}\text { Sculpture } \\
\text { State }\end{array}$ & $\begin{array}{l}\text { Columellae } \\
\text { State }\end{array}$ & $\begin{array}{c}\text { Nexine } \\
\text { State }\end{array}$ \\
\hline $\begin{array}{l}\text { I-Tribe } \\
\text { Gnaphalieae } \\
\text { 1. Unassigned } \\
\text { Gnaphalieae } \\
\text { Phagnalon } \\
\text { barbeyanum }\end{array}$ & II & P.Sh. & Wide & Tapered & La., rect. & Spines & Perforate & $3+4$ & 1 & + \\
\hline P. nitidum & II & O.Sh. & Wide & Pointed & Lo., ovate & Spinules & Perforate & 6 & 1 & ++ \\
\hline P. rupestre & II & P.Sh. & Wide & Tapered & Lo.,ovate. & Spines & Perforate & 6 & 1 & + \\
\hline $\begin{array}{c}\text { P. schweinfurthii } \\
\text { 2.Subtribe } \\
\text { Gnaphaliinae }\end{array}$ & II & O.Sh. & Wide & Pointed & Lo.,ovate & Spines & Perforate & 6 & 1 & ++ \\
\hline Filago contracta & $I$ & Sh. & \pm Wide & Pointed & Lo. ellep. & Spinules & Perforate & 5 & 1 & + \\
\hline F. desertorum & $I$ & Sh. & \pm Wide & Pointed & Lo., ovate & Spinules & Perforate & 6 & 1 & + \\
\hline F. mareotica & $I$ & P.Sh. & \pm Wide & Pointed & Lo. ellep. & Spinules & Perforate & 6 & 1 & + \\
\hline F. prolifera & $I$ & Sh. & \pm Wide & Pointed & Lo., ovate & Spinules & Perforate & 6 & 1 & + \\
\hline $\begin{array}{l}\text { Gnaphalium } \\
\text { uliginosum* }\end{array}$ & $I$ & Sh. & Wide & Tapered & Lo., ovate & Spines & Perforate & $6+2$ & 1 & ++ \\
\hline $\begin{array}{l}\text { Helichrysum } \\
\text { conglobatum }\end{array}$ & II & Sh. & Wide & Tapered & Lo., ovate & Spinules & Perforate & 6 & 1 & + \\
\hline H. glumaceum & II & Sh. & Wide & Pointed & La., rect & Spines & Perforate & $6+3$ & 1 & + \\
\hline H. orientale & II & O.Sh. & Wide & Tapered & Circular & Spines & Perforate & 3 & 1 & + \\
\hline $\begin{array}{l}\text { Homognaphalium } \\
\text { pulvinatum }\end{array}$ & $I$ & Sh. & Wide & Pointed & Circular & Spinules & Perforate & 6 & 1 & + \\
\hline Ifloga spicata & II & Sh. & \pm Wide & Tapered & Lo. ellep. & Spinules & Perforate & 6 & 1 & ++ \\
\hline $\begin{array}{c}\text { Lasiopogon } \\
\text { muscoides }\end{array}$ & $I$ & Sh. & Slender & Tapered & Lo. ellep. & Spinules & Perforate & 6 & 1 & ++ \\
\hline $\begin{array}{l}\text { Pseudognaphalium } \\
\text { luteoalbum } \\
\text { II-Tribe Helenieae } \\
\text { 1.Subtribe } \\
\text { Flaveriinae }\end{array}$ & $I$ & Sh. & Wide & Pointed & Lo., ovate & Spinules & Perforate & 6 & 1 & + \\
\hline $\begin{array}{c}\text { Flaveria bidentis } \\
\text { 2.Subtribe } \\
\text { Pectidinae }\end{array}$ & II & Subob & Wide & Pointed & Lo., ovate & Spines & Perforate & 3 & 1 & + \\
\hline Tagetes minuta & II & Sh. & Wide & Pointed & La., rect & Spines & Perforate & 3 & 1 & + \\
\hline $\begin{array}{l}\text { III-Tribe Plucheeae } \\
\text { Sphaeranthus } \\
\text { suaveolens** } \\
\text { IV-Tribe } \\
\text { Senecioneae } \\
\text { 1.Subtribe } \\
\text { Senecioninae }\end{array}$ & II & Sh. & Wide & Tapered & Circular & Spines & Perforate & 2 & 1 & ++ \\
\hline Senecio aegyptius & II & O.Sh. & Narrow & Pointed & La., rect & Spines & Perforate & 3 & 1 & ++ \\
\hline S. flavus & II & Sh. & Wide & Pointed & Lo., ovate & Spines & Perforate & 3 & 1 & ++ \\
\hline $\begin{array}{l}\text { S. glaucus subsp. } \\
\text { coronopifolius }\end{array}$ & II & Sh. & \pm Wide & Pointed & Lo., ovate & Spinules & Perforate & 6 & 1 & ++ \\
\hline $\begin{array}{l}\text { S. glaucus subsp. } \\
\text { glaucus }\end{array}$ & II & O.Sh. & \pm Wide & Pointed & Lo. ellep. & Spines & Perforate & 6 & 1 & ++ \\
\hline S. hoggariensis & II & O.Sh. & Slender & Tapered & Lo., ovate & Spines & Perforate & 6 & 1 & ++ \\
\hline S. vulgaris & II & Subob & \pm Wide & Tapered & Lo. ellep. & Spines & Perforate & 5 & 1 & ++ \\
\hline
\end{tabular}

Col. wid. at eq. $=$ Colpi Width at equator, Ellep. = Elleptic, La. = Lalongate, Lo. = Lolongate, O. = Oblate, P. = Prolate, Rect. = Rectangular, Sh. = Spheroidal, Subob. $=$ Suboblate, \pm Wide $=$ Slightly wide, $\mathrm{I}=$ Pollen 3 -zonocolporate, $\mathrm{II}=$ Pollen 3 -zonocolpororate, $1=$ Columellae with thin, short and unbranched rods, + $=$ Sexine as thick as nexine, $++=$ Sexine thicker than nexine, $2=$ Sculpturing diameter increases towards the aperture margins, $3=$ Sculpturing diameter increases towards the echinae bases, $4=$ Sculpturing diameter increases towards the pollen pole, $5=$ Sculpturing restrict to the echinae bases, $6=$ Sculpturing diameter the same on the whole of the pollen surface, $*=$ Aperture margins perforate, $* *=$ Aperture margins microreticulate. 
Key to taxa of Filago pollen type

1.a. Pollen prolate-spheroidal in shape..Filago mareotica

b. Pollen sheroidal in shape...

2.a. Colpi slender at equator......Lasiopogon muscoides

b. Colpi otherwise................................. 3

3.a. Colpi wide at equator......Gnaphalium uliginosum ..Homognaphalium pulvinatum Pseudognaphaliumluteoalbum

b. Colpi slightly wide at equator.........Filago contracta .F. desertorum F. prolifera

2. Senecio pollen type

LM (Figures 1(a)-(h), Figures 2(c)-(h), (k), (l), Figures 3(a)-(p), Figures 4(a), (b), SEM Figures 4(c)-(h), Figures 5(a)-(d), (j)-(o), Figures 6(a)-(j).

Pollen grains 3-colpororate, spheroidal, oblate-spheroidal, prolate-spheroidal to suboblate $(\mathrm{P} / \mathrm{E}=0.85-1.10)$, $21-44(20-45) \times 21-44(20-45) \mu \mathrm{m}$. Apocolpium diameter 5-16 (3-18) $\mu \mathrm{m}$. Colpi 12-27 (10-30) $\mu \mathrm{m}$ long, 3-5 (2-7) $\mu \mathrm{m}$ wide, wide, slightly wide, slender or narrow at the equator, pointed-tapered towards the ends. Mesocolpium 10-24 (8-25) $\mu \mathrm{m}$ wide. Ora lolongate-lalongate (circular in both Helichrysum orientale and Sphaeranthus suaveolens), with ovate, rectangular to elleptic-shaped, 4-8 (3-10) $\mu \mathrm{m}$ in diameter. Exine 3-8 (2-9) $\mu \mathrm{m}$ thick (spines included) at centre of mesocolpia, with the same thickness towards the aperture margins. Sexine thicker than nexine or as thick as nexine, tectate, with long, pointed spines or spinules, 2-5 (1-6) $\mu \mathrm{m}$ high, perforated; perforations number and diameter the same on the whole of the pollen surface or increase towards the echinae bases (increase towards the aperature margins in Sphaeranthus suaveolens or restrict to the echinae bases in Senecio vulgaris); the intratectal elements thin, minute, radial and supported by fairly thin and short infratectal columellae. Nexine 1-3 (1-4) $\mu \mathrm{m}$ thick, increasing in thickness towards the aperture margins.

The following taxa belong to this type:

I-Tribe Gnaphalieae

Gnaphalieae unassigned to Subtribe.

Genus Phagnalon Cass (4 species).

Species included:

1. P. barbeyanum Asch. \& Schweinf., Mém. Inst. Egypt. 2: 87 (1887). (1888).

Syn. P. Aegyptiacum Boiss., Fl. Orient. Suppl. 296

2. P. nitidum Fresen., Mus. Senckenb. 3: 81 (1839).

3. P. rupestre ( L.) DC., Prodr. 5: 396 (1836).

Syn. Conyza rupestris L., Mant. 113 (1767).

4. P. schweinfurthii Sch. Bip. ex Schweinf., Verh. K.

K. Zool. Bot. Ges. Wien 18: 685 (1868).

Subtribe Gnaphaliinae Less.
Genus Helichrysum Mill. Syn. Leontonyx Cass. (3 species).

Species included:

5. H. conglobatum (Viv.) Steud., Nomencl. Bot., ed. 2, 1: 738 (1840).

Syns. Gnaphalium conglobatum Viv., Fl. Libyc. Spec. 55, t. 3, f. 5 (1824).

Helichrysum siculum Boiss., Fl. Orient. 3: 229 (1875).

6. H. glumaceum DC., Prodr. 6: 197 (1838).

Syn. Achyrocline glumacea (DC.) Oliv. \& Hiern in Oliv., Fl. Trop. Afr. 3: 340 (1877)

7. H. orientale (L.) Gaertn., Fruct. Sem. Pl. 2: 404 (1791). (1753).

Syn. Gnaphalium orientale L., Sp. Pl., ed. 1, 853

Genus Ifloga Cass. Syn. Comptonanthus B. Nord (one species).

Species included:

8. I. spicata (Forssk.) Sch.Bip. in Webb \& Berthel., Phyt. Canar. 2: 310 (1845).

Syns. Chrysocoma spicata Forssk., Fl. Aegypt.-Arab. LXXIII (1775).

Chrysocoma spicatum (Forssk.) Vahl, Symb. Bot. 1: 70 (1790).

II. Tribe Helenieae Benth.

Subtribe Flaveriinae Less.

Genus Flaveria Juss. (one species).

Species included:

9. F. bidentis (L.) Kuntze, Rev. Gen. 3: 148 (1898).

Syns. Ethulia bidentis L., Mant. 110 (1767).

Flaveria contrayerba (Cav.) Pers., Syn. Pl. 2: 489 (1807).

Flaveria bonariensis DC., Prodr. 5: 635 (1836)

Subtribe Pectidinae Less.

Genus Tagetes L. (one species).

Species included:

10. T. minuta L., Sp. Pl., ed. 1, 887 (1753).

III. Tribe Plucheeae Anderb.

Genus Sphaeranthus L. (one species).

Species included:

11. S. suaveolens (Forssk.) Dc., Prodr. 5: 370 (1836).

Syn. Polycephalos suaveolens Forssk., Fl. Aegypt.-Arab. 154 (1775).

IV. Tribe Senecioneae

Subtribe Senecioninae Dumort.

Genus Senecio L. (6 species).

Species included:

12. S. aegyptius L., Sp. Pl., ed. 1, 867 (1753).

13. S. flavus (Decne.) Sch. Bip. In Webb \& Berthel., Phyt. Canar. 3: 317 (1847).

Syns. Crassocephalumflavum Decne., Ann. Sci. Nat. 


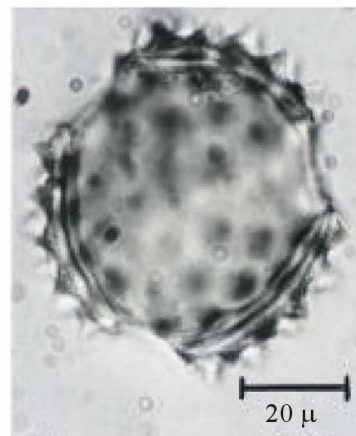

(a)

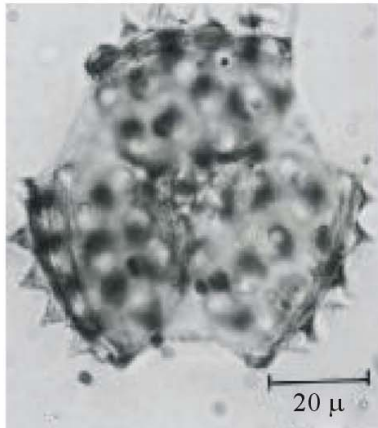

(e)

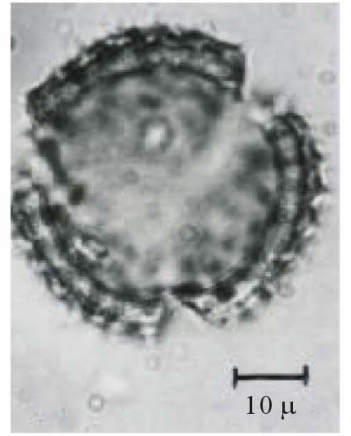

(i)

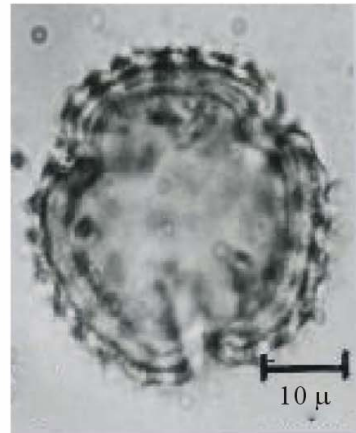

(m)

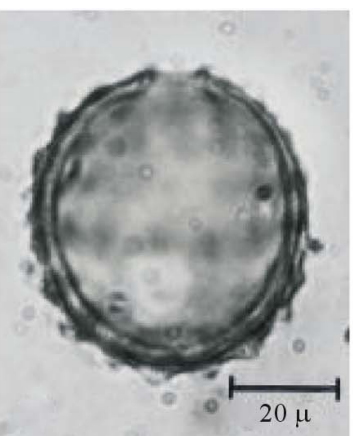

(b)

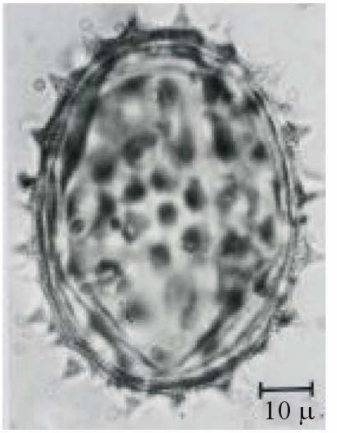

(f)

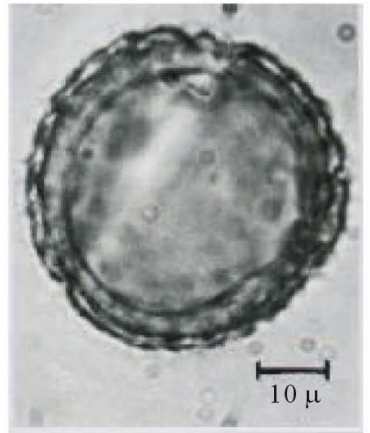

(j)

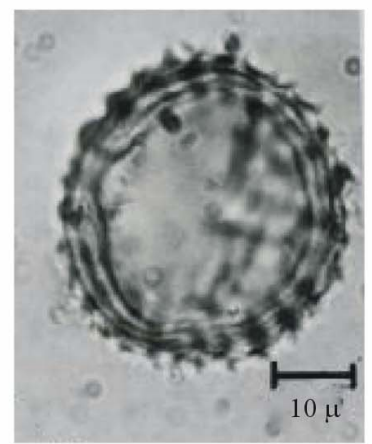

(n)

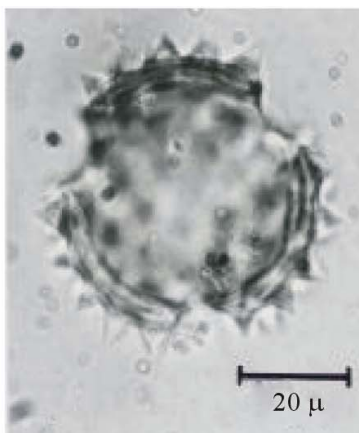

(c)

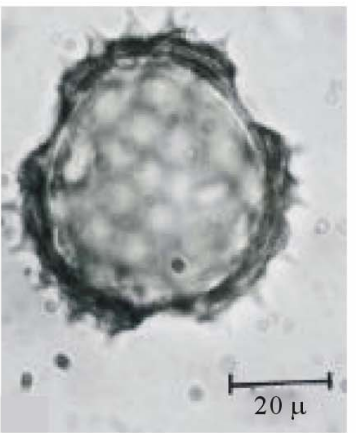

(g)

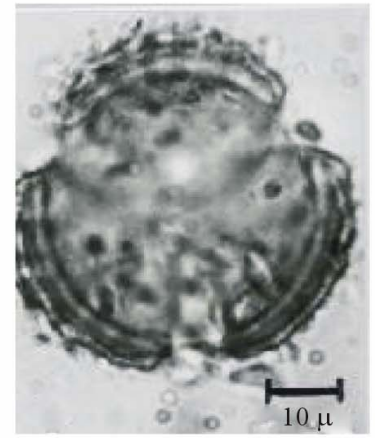

(k)

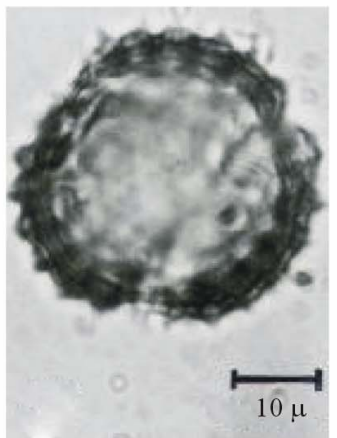

(o)

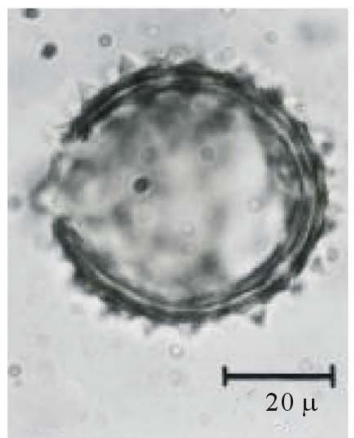

(d)

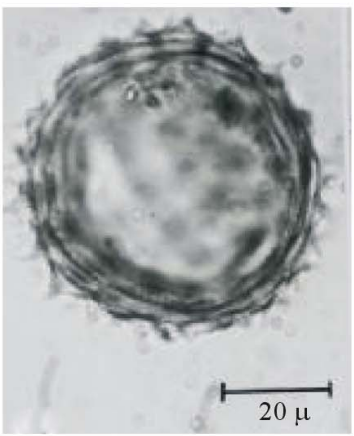

(h)

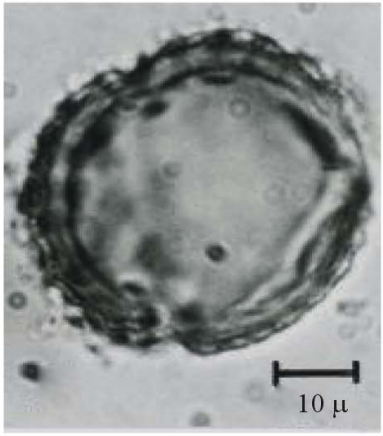

(1)

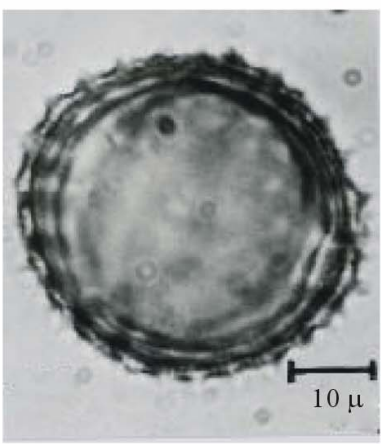

(p)

Figure 1. LM observations of acetolysed pollen grains (LM $\times=1000)$. (a)-(b) Phagnalon barbeyanum, (a) Oblique polar view, (b) Oblique equatorial view. (c)-(d) P. nitidum, (c) Polar view, (d) Oblique equatorial view. (e)-(f) P. rupestre, (e) Polar view, (f) Equatorial view. (g)-(h) P. schweinfurthii, (g) Polar view, (h) Equatorial view. (i)-(j) Filago contracta, (i) Polar view, (j) Equatorial view. (k)-(l) F. desertorum, (k) Polar view, (k) Oblique equatorial view. (m)-(n) F. mareotica, (m) Polar view, (n) Equatorial view. (o)-(p) F. prolifera, (o) Oblique polar view, (p) Equatorial view. 

of Compositae from Egypt

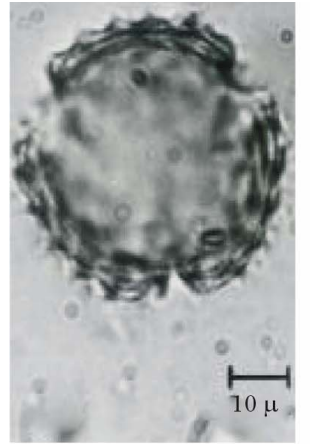

(a)

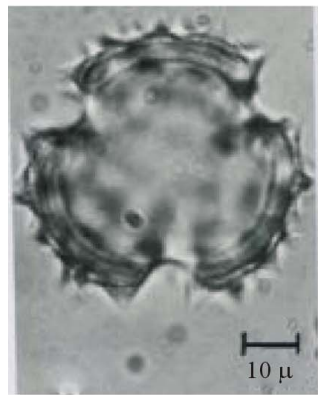

(e)

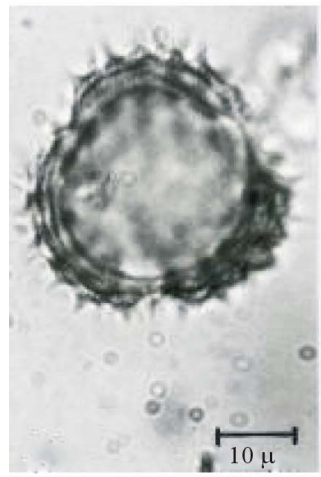

(i)

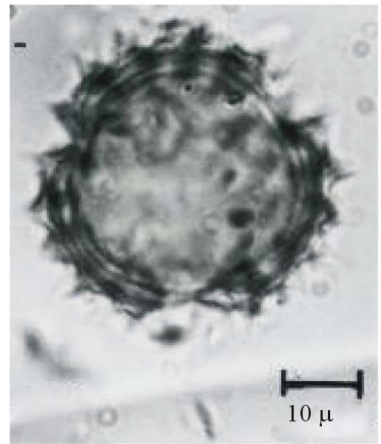

(m)

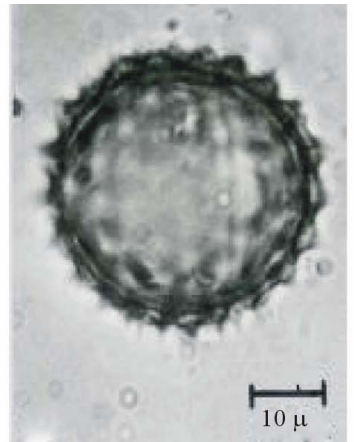

(b)

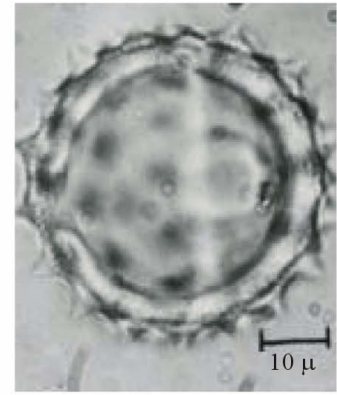

(f)

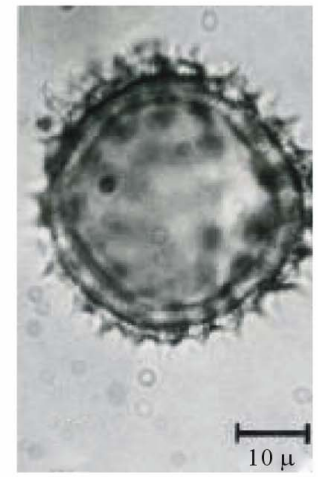

(j)

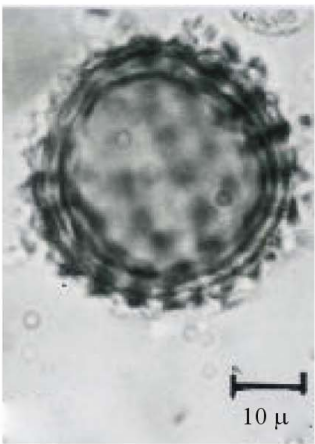

(n)

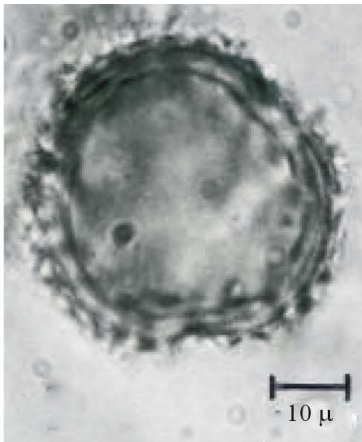

(c)

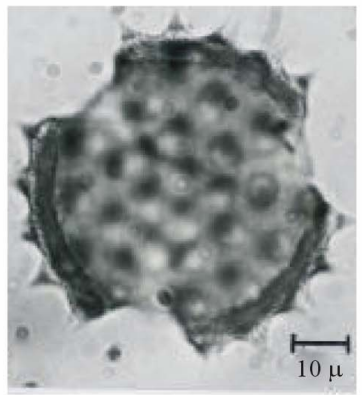

(g)

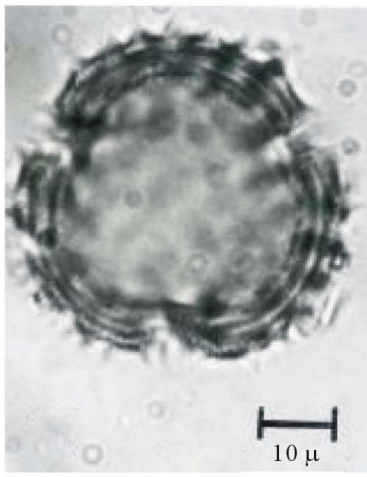

(k)

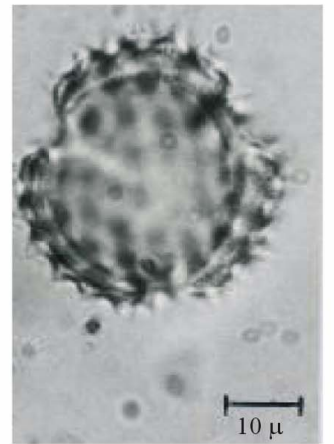

(o)

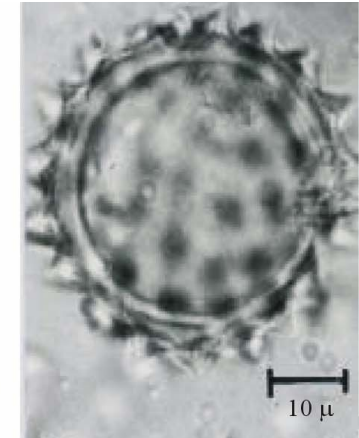

(d)

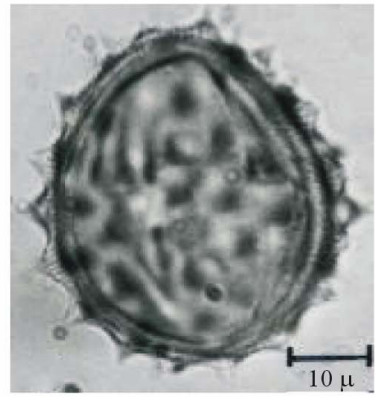

(h)

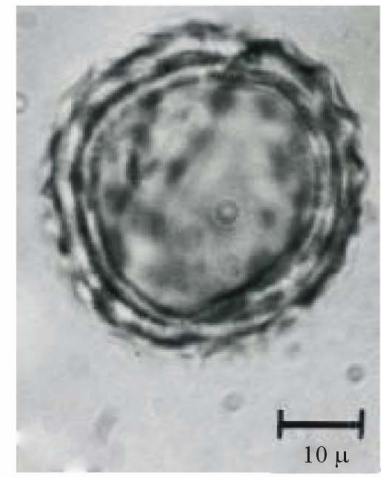

(1)

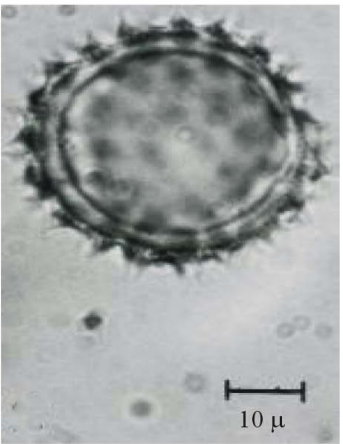

(p)

Figure 2. LM observations of acetolysed pollen grains (LM $\times=1000)$. (a)-(b) Gnaphalium uliginosum, (a) Polar view, (b) Equatorial view. (c)-(d) Helichrysum conglobatum, (c) Polar view, (d) Equatorial view. (e)-(f) H. glumaceum, (e) Polar view, (f) Equatorial view. (g)-(h) H. orientale, (g) Polar view, (h) Equatorial view. (i)-(j) Homognaphalium pulvinatum, (i) Polar view, (j) Equatorial view. (k)-(l) Ifloga spicata, (k) Polar view, (l) Equatorial view. (m)-(n) Lasiopogon muscoides, (m) Polar view, (n) Equatorial view. (o)-(p) Pseudognaphaliumluteoalbum, (o) Polar view, (p) Equatorial view. 


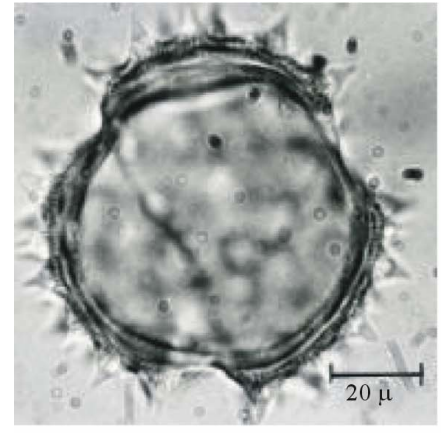

(a)

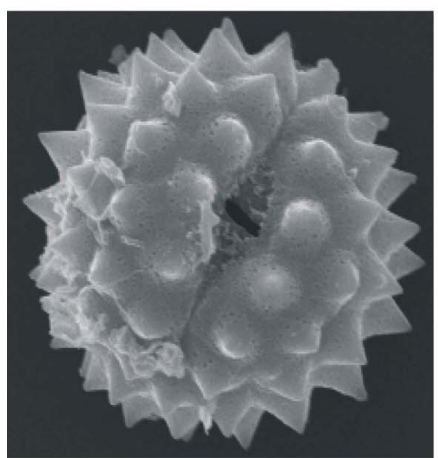

(d)

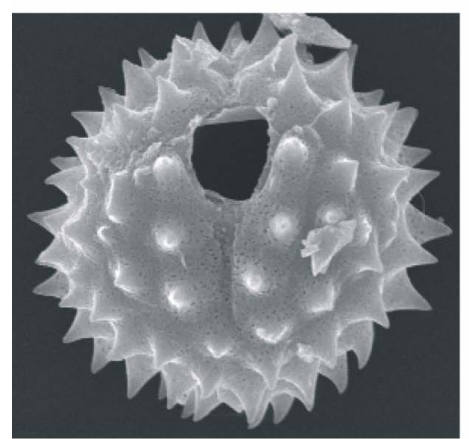

(g)

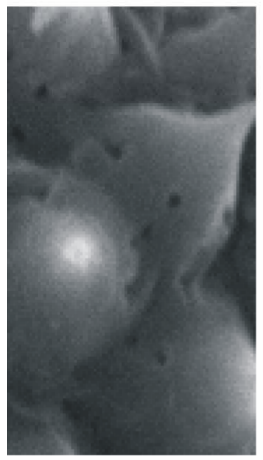

(j)

(k)

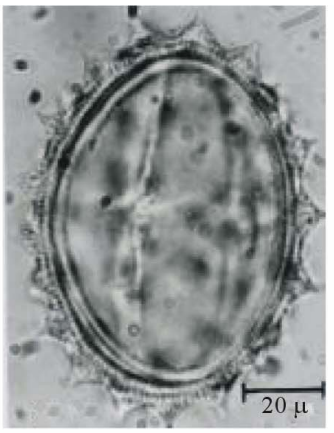

(b)

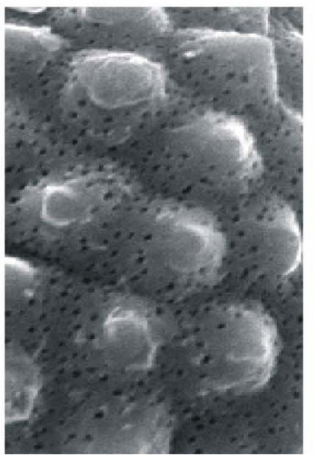

(e)

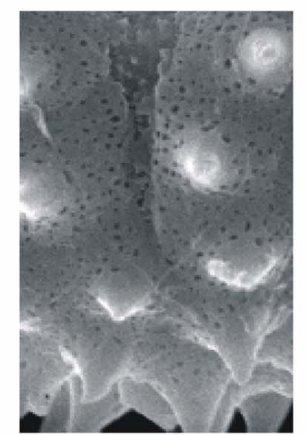

(h)

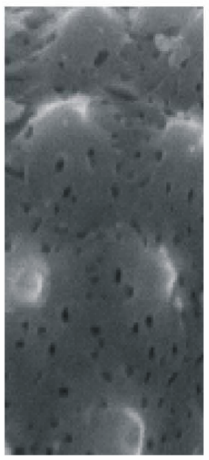

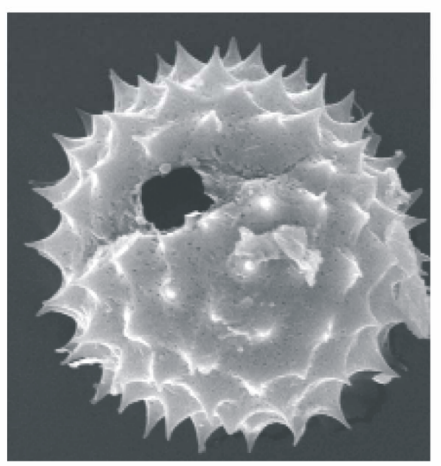

(1)

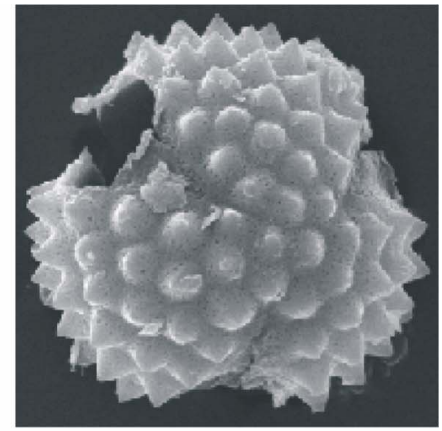

(c)

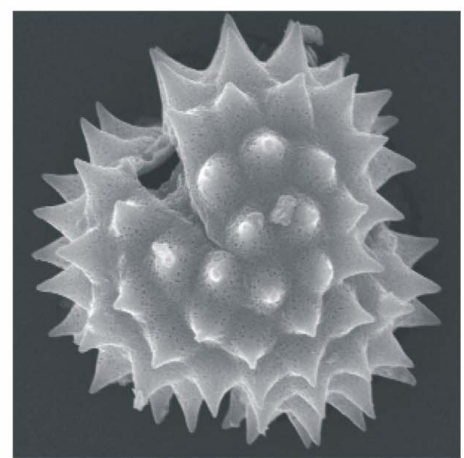

(f)

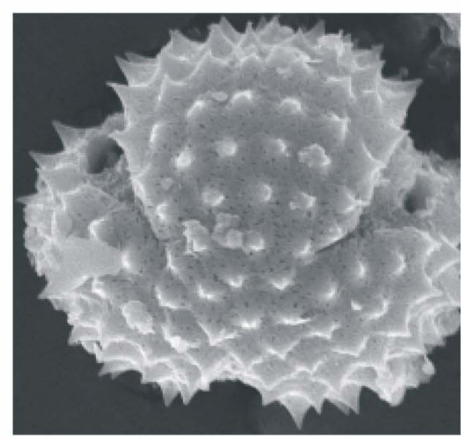

(i)

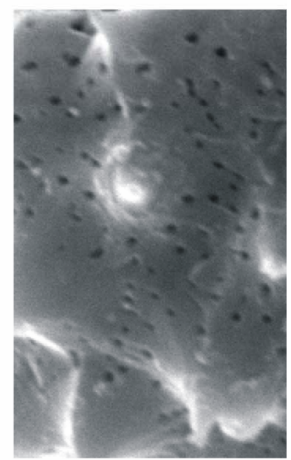

(m)

Figure 3. LM and SEM observations of acetolysed pollen grains (LM $\times=1000$, SEM $\times=5000$ - 15000). (a)-(b) Senecio vulgaris, (a) Oblique polar view, (b) Equatorial view. (c)-(e) Phagnalon barbeyanum, (c) Polar view, (d) Equatorial view, (e) Magnified part of exine. (f)-(h) Phagnalon rupestre, (f) Oblique polar view, (g) Oblique equatorial view, (h) Magnified part of exine. (j) Filago contracta, (i) Magnified part of exine. (i) (k) Filago prolifera, (i) Oblique polar view, (k) Magnified part of exine, (l)-(m) Gnaphalium uliginosum, (l) Oblique equatorial view, (m) Magnified part of exine. 


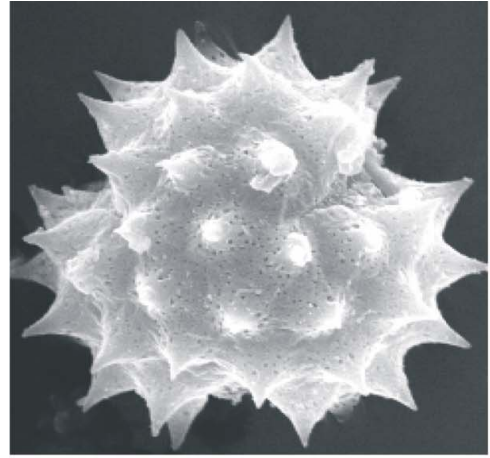

(a)

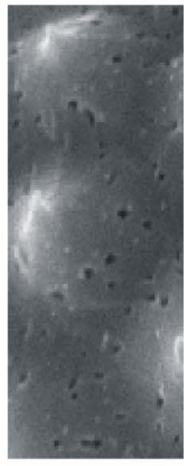

(d)

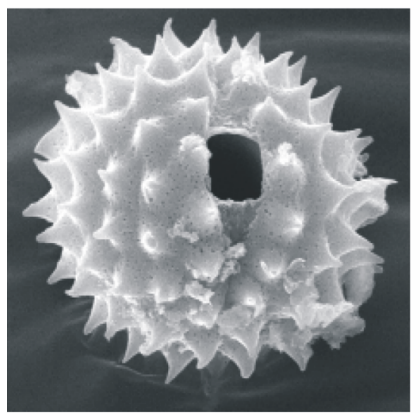

(h)

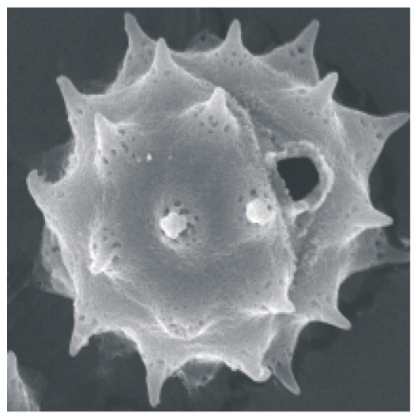

(1)

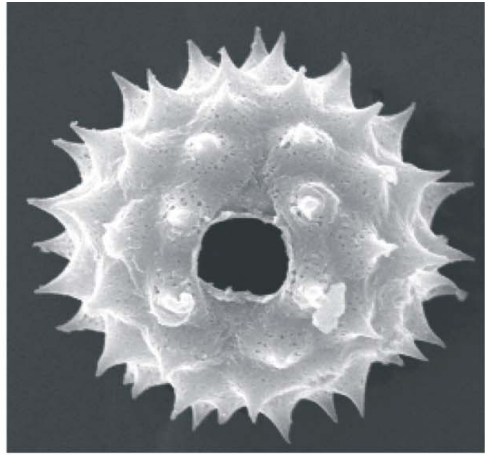

(b)

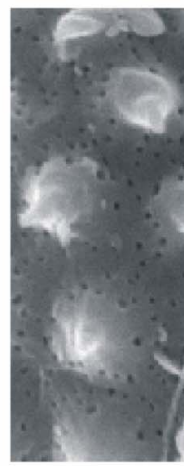

(f)

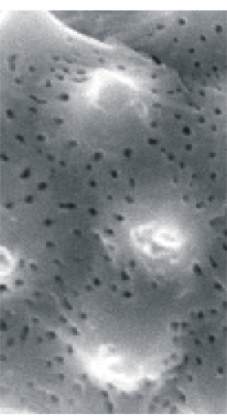

(i)

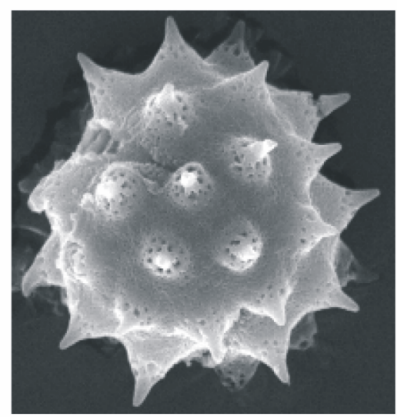

(j)

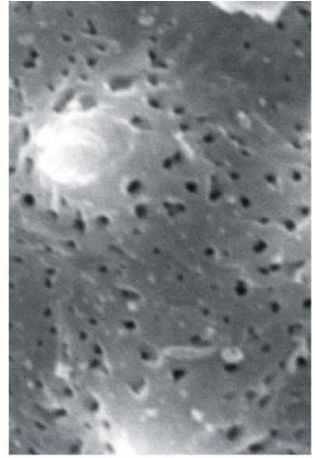

(c)

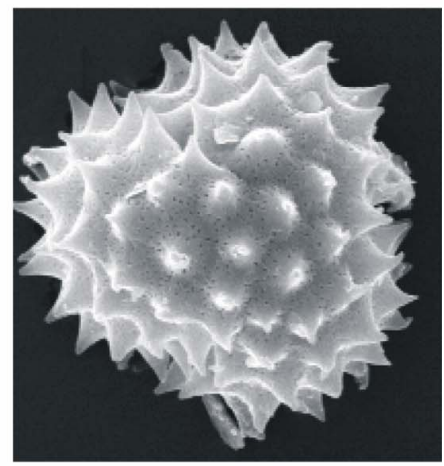

(g)

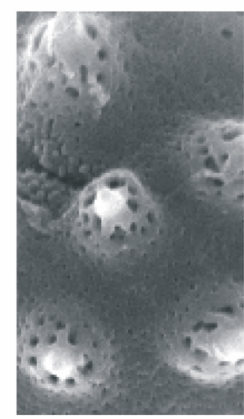

$(\mathrm{k})$

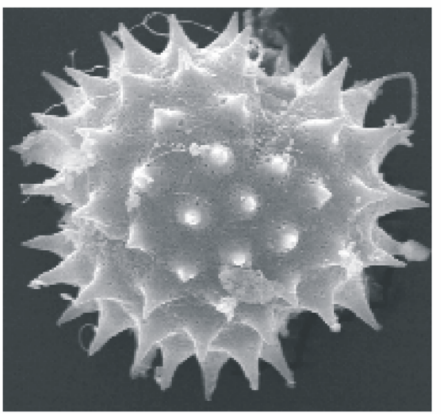

$(\mathrm{m})$

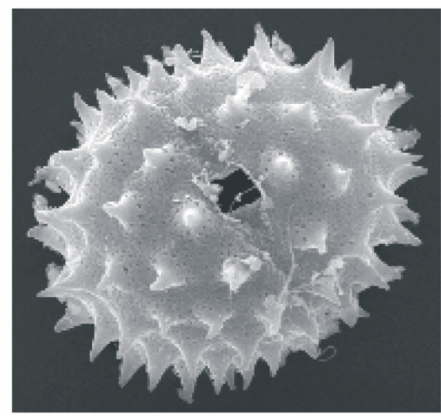

(n)

Figure 4. SEM observations of acetolyzed pollen grains (SEM $\times$ 5000-15000). (a)-(c) Helichrysum orientale, (a) Polar view, (b) Equatorial view, (c) Magnified part of exine. (d) Ifloga spicata, (d) Magnified part of exine. (e)-(f) Lasiopogon muscoides, (e) Oblique equatorial view, (f) Magnified part of exine. (g)-(i) Pseudognaphalium luteoalbum, (g) Oblique polar view, (h) Equatorial view, (i) Magnified part of exine. (j)-(l) Flaveria bidentis, (j) Oblique polar view, (k) Oblique equatorial view, (l) Magnified part of exine. (m)-(n) Tagetes minuta, (m) Polar view, (n) Equatorial view. 


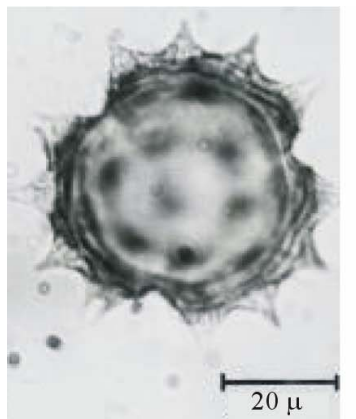

(a)

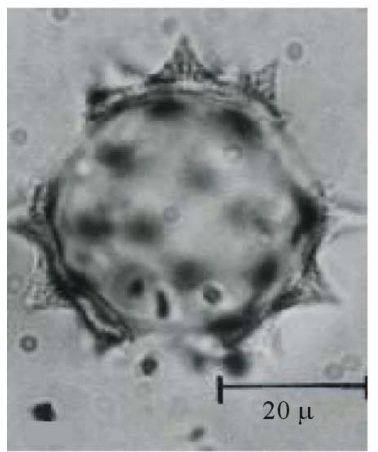

(e)

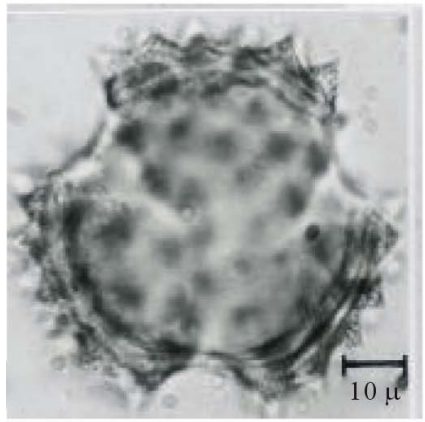

(i)

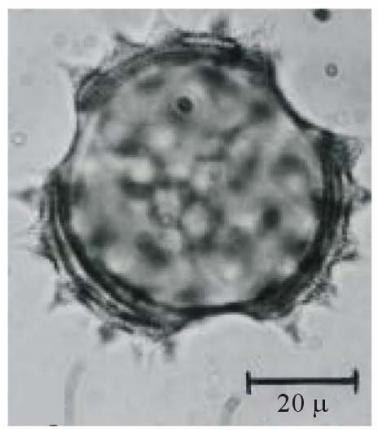

(m)

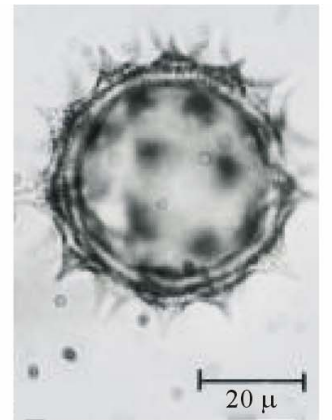

(b)

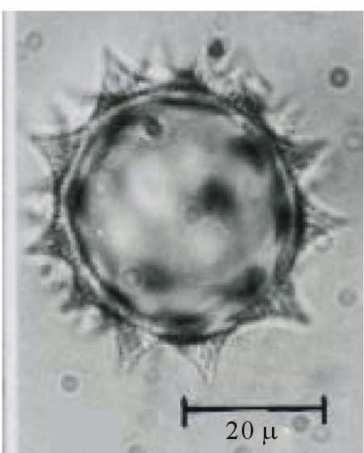

(f)

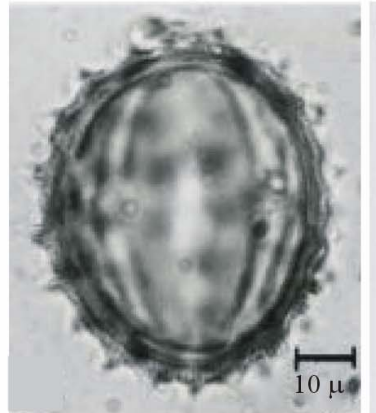

(j)

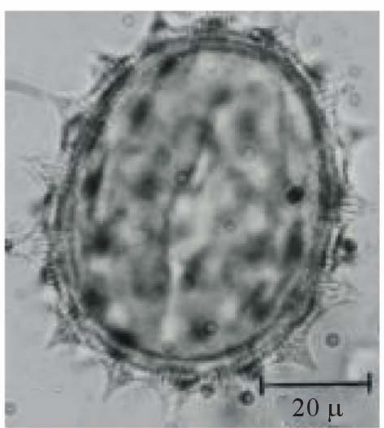

(n)

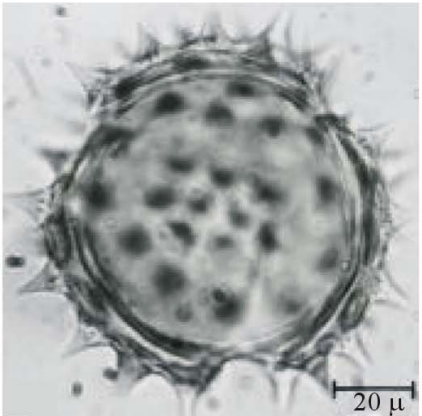

(c)

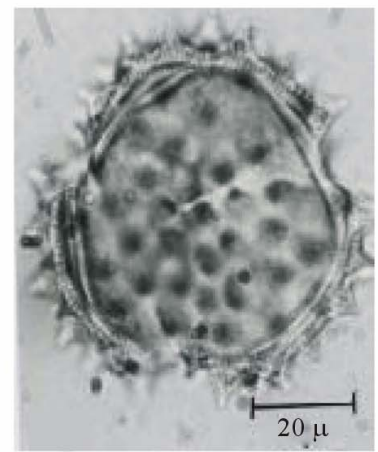

(g)

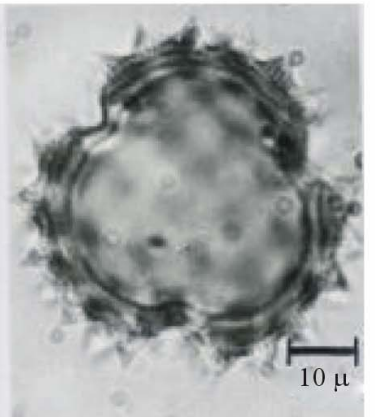

(k)

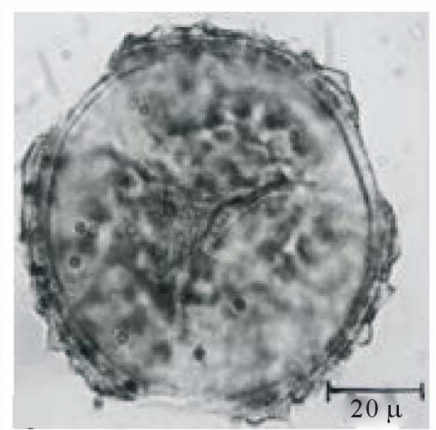

(o)

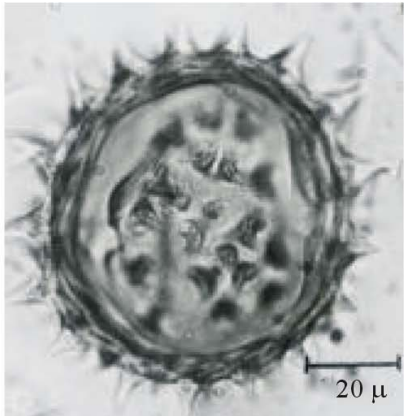

(d)

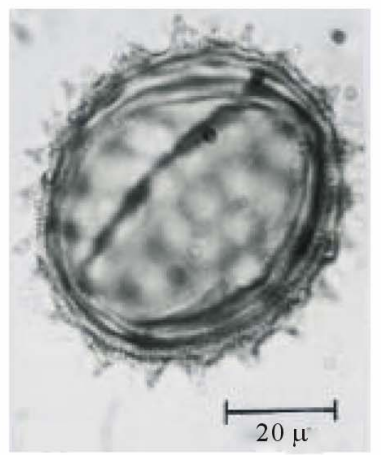

(h)

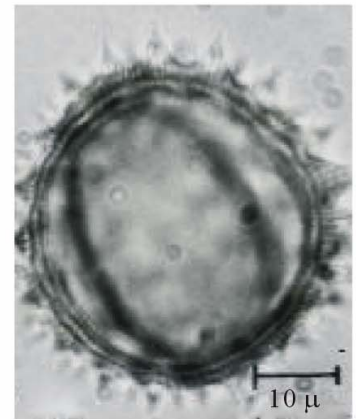

(1)

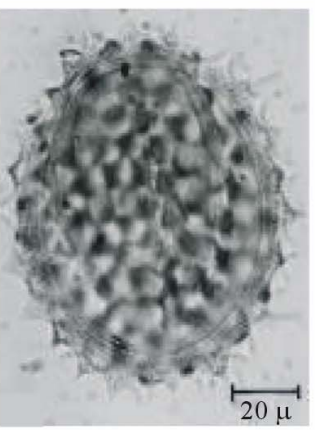

(p)

Figure 5. LM observations of acetolysed pollen grains (LM $\times=1000)$. (a)-(b) Flaveria bidentis, (a) Polar view, (d) Equatorial view. (c)-(d) Tagetes minuta, (c) Polar view, (d) Equatorial view. (e)-(f) Sphaeranthus suaveolens, (e) Polar view, (f) Equatorial view. (g)-(h) Senecio aegyptius, (g) Polar view, (h) Equatorial view. (i)-(j) S. flavus, (i) Polar view, (j) Equatorial view. (k)-(l) $S$. glaucus subsp. coronopifolius, (k) Polar view, (l) Equatorial view. (m)-(n) S. glaucus subsp. glaucus, (m) Polar view, (n) Equatorial view. (o)-(p) S. hoggariensis, (o) Polar view, (p) Equatorial view. 


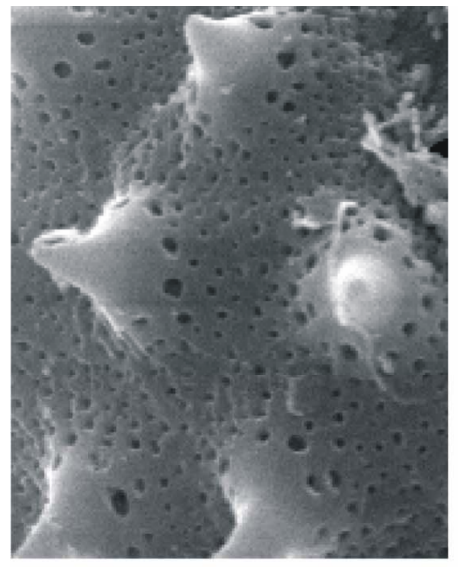

(a)

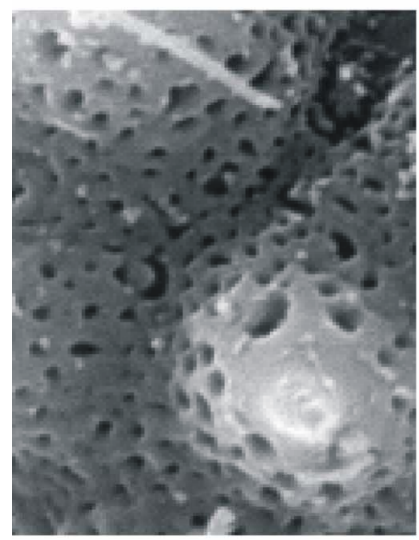

(d)

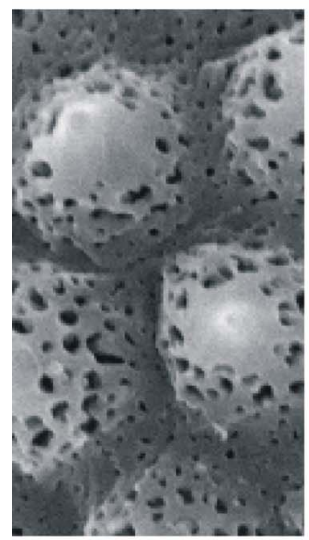

(g)

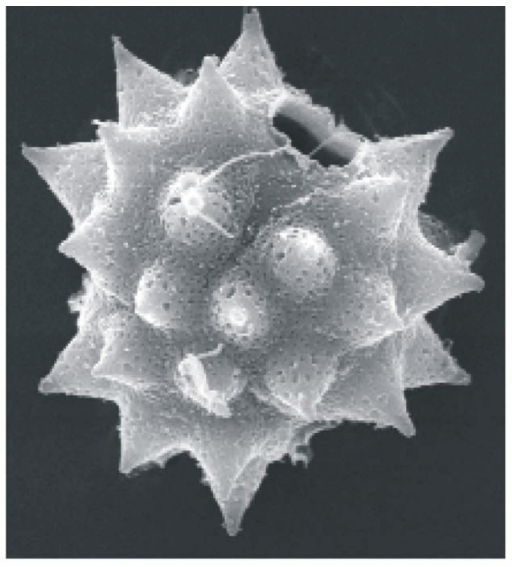

(b)

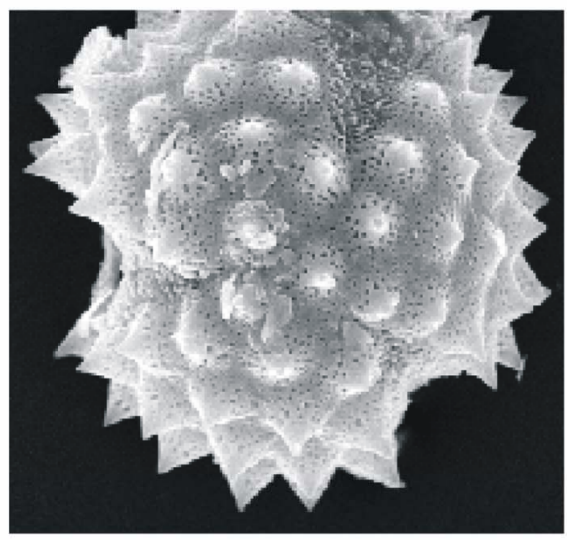

(e)

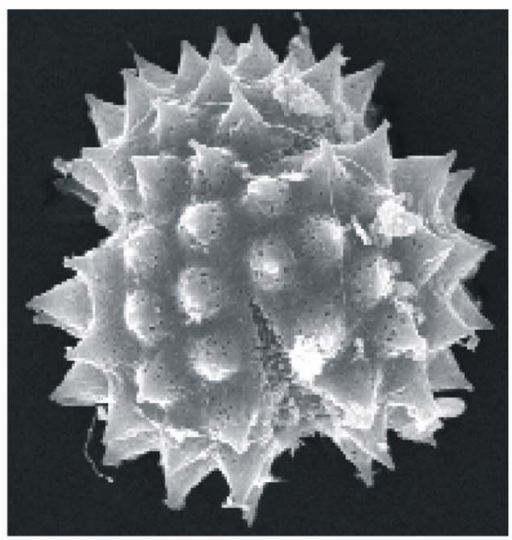

(h)

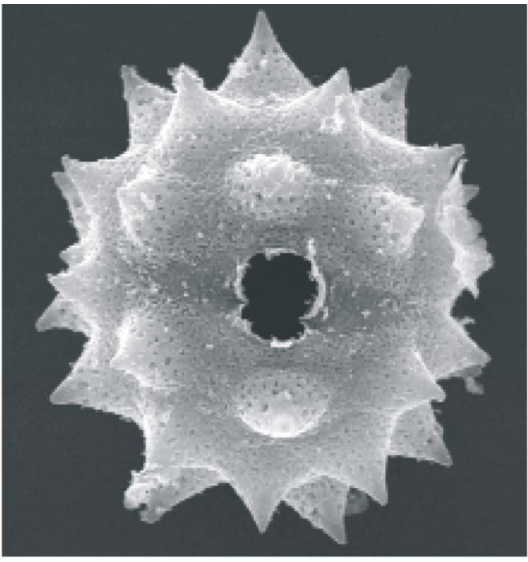

(c)

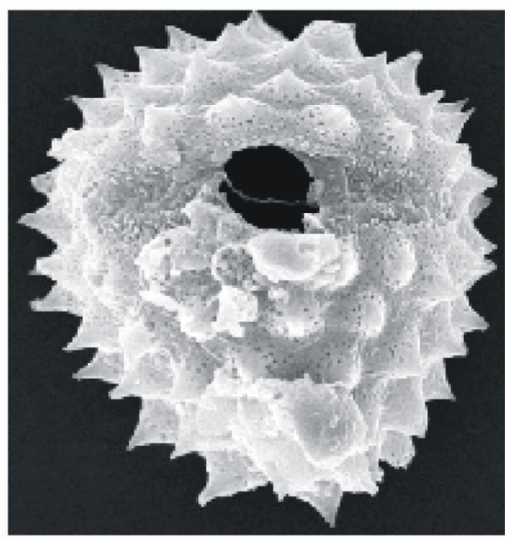

(f)

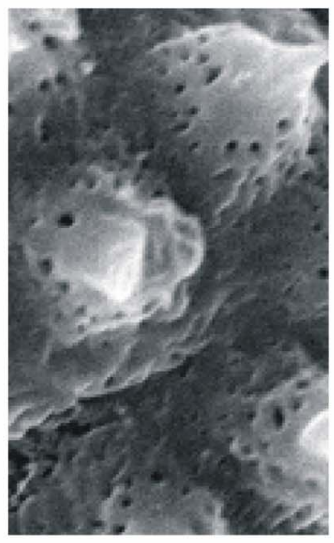

(i)

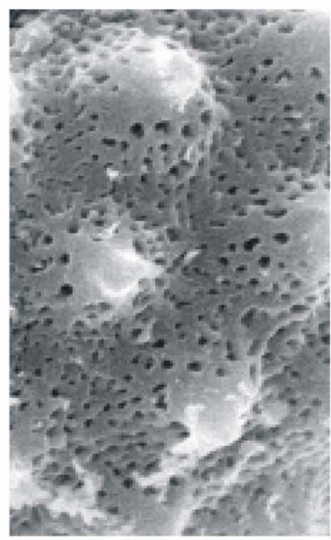

(j)

Figure 6. SEM observations of acetolyzed pollen grains (SEM $\times$ 5000-15000). (a) Tagetes minuta, (a) Magnified part of exine. (b)-(d) Sphaeranthus suaveolens, (b) Oblique polar view, (c) Equatorial view, (d) Magnified part of exine. (e)-(g) Senecio flavus, (e) Polar view, (f) Equatorial view, (g) Magnified part of exine. (h)-(i) Senecio vulgaris, (h) Polar view, (i) Magnified part of exine. (j) Senecio aegyptius, (j) Magnified part of exine.

Bot., sér. 2, 2: 265 (1834).

Senecio decaisnei DC., Prodr. 6: 342 (1838).

14. S. glaucus L., Sp. Pl., ed. 1, 868 (1753). subsp. coronopifolius (Maire) C. Alexander, Notes Roy. Bot. Gard. Edinb. 37: 412 (1979).

Syns. Senecio coronopifolius Desf., Fl. Atlant. 2: 
273 (1799), nom. illeg., non Burm.f. (1768).

Senecio desfontainei Druce, Brit. Pl. List, ed. 2, 61 (1928).

15. S. glaucus L., Sp. Pl., ed. 1, 868 (1753). subsp. glaucus

Syns. Senecio vernalis Waldst. \& Kit. var. carnosus

Post. Fl. Syr. Pal. Sinia, ed. 1, 442 (1896).

Senecio joppensis Dinsm. In Post, Fl. Syr. Pal. Sinai. Ed. 2, 2: 69 (1933).

16. S. hoggariensis Batt. \& Trab., Bull. Soc. Bot. France 58: 671 (1911).

17. S. vulgaris L., Sp. P1., ed. 1, 867 (1753).

Key to taxa of Senecio pollen type

1.a. Polar area long axis median $21-29 \mu \mathrm{m} . . . \ldots \ldots \ldots . . .4$

b. Polar area long axis median $31-44 \mu \mathrm{m} . \ldots \ldots \ldots \ldots \ldots . . .2$

2.a. Polar area long axis $44(42$ - 45) $\mu \mathrm{m}$...Tagetes minuta

b. Polar area long axis median $31-37 \mu \mathrm{m}$.

....3

3.a. Polar area long axis 31 - $32 \mu \mathrm{m}$...Phagnalon rupestre Senecio aegyptius

S. glaucus subsp. glaucus

b. Polar area long axis 35 - $37 \mu \mathrm{m}$...Senecio hoggariensis S. vulgaris

4.a. Polar area long axis $21-25 \mu \mathrm{m}$....Phagnalon nitidum Helichrysum conglobatum Ifloga spicata

Sphaeranthus suaveolens

................. Senecio glaucus subsp. coronopifolius

b. Polar area long axis $26-29 \mu \mathrm{m}$.

Phagnalon barbeyanum

P. schweinfurthii

Helichrysum glumaceum H. orientale

Flaveria bidentis Senecio flavus

\section{Discussion}

Palynological characters applied to the species of Gnaphalieae, Helenieae, Plucheeae and Senecioneae in Egypt proved to be useful in the distinction between two groups. The pollen grains are usually 3-zonocolporate or 3-zonocolpororate with lolongate or lalongate (rarely circular) ora.

There is semi-similarity between the pollen grains of the investigated taxa, as a result it will be difficult to make accurate distinguishing and to construct true key between the studied species. Two pollen types are distinguished according to the pollen class. The Senecio pollen type, in which the pollen are 3-colpororate, is characteristic of the species of genera Phagnalon, Helichrysum, Ifloga, Flaveria, Tagetes, Sphaeranthus and senecio. The Filago pollen type, in which the pollen are 3 -colporate, is characteristic of the species of genera
Filago, Gnaphalium, Homognaphalium, Lasiopogon and Pseudognaphalium.

The smallest pollen grains are those of Lasiopogon muscoides species $20(19-23) \mu \mathrm{m}$ and the largest are those of Tagetes minuta 44 (42-45) $\mu \mathrm{m}$. Pollen size of other taxa ranged between (21-37) $\mu \mathrm{m}$. Pollen grains are more or less similar in shape being spheroidal, oblate-spher- oidal, prolate-spheroidal to suboblate.

Ora shape contributes to differentiate between the related species of Helichrysum glumaceum, Ifloga spicata, Flaveria bidentis, Tagetes minuta, Senecio flavus and Senecio vulgaris are characterized by lalongate ora shape, Helichrysum orientale, Homognaphalium pulvinatum and Sphaeranthus suaveolens exhibit circular ora shape, while lolongate ora shape is observed in the remaining studied species. The aperture width also contributes to differentiate all studied species of genus Filago, Ifloga spicata, Senecio glaucus subsp. coronopifolius, S. glaucus subsp. Glaucus and S. vulgaris which exhibit colpi are slightly width at equator, Lasiopogon muscoides and $S$. hoggariensis which have slender colpi at equator, Senecio aegyptius has narrow colpi at equator from the other investigated taxa which are characterized by wide colpi at equator. It also points to the close relationship between the four Filago species which exhibit very close pollen grains being similar in pollen shape, colpi characters, sculpture and dimensions.

The pollen sculpture is more uniform in most investigated species with perforate texture. The pollen grains are spiny to spinulate with perforate echinae bases. In Gnaphalium uliginosum aperture margins are perforate while in Sphaeranthus suaveolens species they are microreticulate, also in both Filago contracta and Senecio vulgaris perforation restrict to the echinae bases.

\section{REFERENCES}

[1] L. Boulos, "Flora of Egypt, Verbenaceae-Compositae," Al-Hadara Publishing, Cairo, Vol. 3, 2002.

[2] J. Muller, "Fossil Pollen Records of Extant Angiosperms," Botanical Review, Vol. 47, No.1, 1981, pp. 1-142. doi:10.1007/BF02860537

[3] J. J. Skvarla, D. A. Larson, "An Electron Microscopic Study of Pollen Morphology in the Compositae with Special Reference to the Ambrosinae," Grana Palynol., Vol. 6, 1965, pp. 210-269. doi:10.1080/00173136509429147

[4] J. J. Skvarla, B. L. Turner, "Systematic Implications from Electron Microscopic Studies of Compositae Pollen-A Review," Annals of the Missouri Botanical Garden, Vol. 53, 1966, pp. 220-256. doi:10.2307/2394944

[5] J. J. Skvarla, B. L. Turner, V. C. Patel and A. S. Tomb, "Pollen Morphology in the Compositae and in Morphologically Related Families," In: V. H. Heywood et al. 
Eds., The Biology and Chemistry of Compositae, Vol. 2, London, 1977, pp. 141-265.

[6] M. S. Zavada, S. E. De Villiers, "Pollen of the Asteraceae from the Paleocene-Eocene of South Africa," Grana, Vol. 39, 2000, pp. 39-45. doi:10.1080/00173130150503795

[7] R. Tormo, J. L. U. Jimenez, "The Apertural System of Pollen Grains in Anthemideae and Cardeae (Compositae) with Special Reference to the Mesoaperture," Review of Palaeobotany and Palynology, Vol. 62, 1990, pp. 1-9.

[8] A. A. Anderberg, "Taxonomy and Phylogeny of the Tribe Gnaphalieae (Asteraceae)," Opera Botanica, Vol. 104, 1991, pp. 1-195.

[9] P. Leins, "Pollensystematische Studien an Inuleen, I: Tarchonanthinae, Plucheinae, Inulinae, Buphthalminae," Botanishe Jahrbücher für Systematik, Pflanzengeschichte und Pflanzengeographie, Vol. 91, 1971, pp. 91-146.

[10] P. Leins, "Pollensystematische Studien an Inuleen, II: Filagininae," Botanishe Jahrbücher für Systematik, Pflanzengeschichte und Pflanzengeographie, Vol. 93, 1973, pp. 603-611.

[11] V. Tackholm, "Students' Flora of Egypt," 2nd Edition,
Cairo University, Cairo, 1974.

[12] M. N. El Hadidi, A. A. Fayed, "Materials for Excursion Flora of Egypt (EFE),” Taeckholmia, Vol. 15, 1994/1995, pp. 96-98.

[13] L. Boulos, "Flora of Egypt Checklist, Revised Annotated Edition," Al-Hadara Pblishing, Cairo, 2009.

[14] K. Bremer, "Asteraceae. Cladistics and Classifications," Timber Press Inc., Portland, 1994.

[15] G. Erdtman, "The Acetolysis Method, a Revised Description," Svensk Bot. Tidskr, Vol. 54, No. 1960, pp. 561-464.

[16] G. Erdtman, "Handbook of Palynology, an Introduction to the Study of Pollen Grains and Spores," Munksgaard, Copenhagen, 1969.

[17] G. Erdtman, "Pollen Morphology and Plant Taxonomy, Angiosperms," Hafner Publishing Co., New York, 1966.

[18] K. Faegri and J. Iversen, "Textbook of Pollen Analysis," 3rd Edition, Munksgaard, Copenhagen.

[19] M. T. Dimon, "Problémes Généraux Soulevés par L'étude Pollinique de Composées Méditerranénnes," Nat. Monsp. Sér. Bot., Vol. 22, 1971, pp. 129-144. 